\title{
Dog bites
}

\author{
Marina Morgan, ${ }^{1}$ John Palmer ${ }^{2}$
}

Old Pathology Laboratory, Royal Devon and Exeter Foundation Trust, Exeter EX25AD

${ }^{2}$ Department of Plastic and

Reconstructive Surgery, Royal Devon and Exeter Foundation

Trust, Exeter

Correspondence to: M Morgan

marina.morgan@rdeft.nhs.uk

BMJ 2007:334:413-7

doi: 10.1136/bmj.39105.659919.BE
Bites and maulings by dogs, sometimes fatal, are a worldwide problem and particularly affect children. Every year 250000 people who have been bitten by dogs attend minor injuries and emergency units in the United Kingdom, ${ }^{1}$ and some of them are admitted to hospital for surgical debridement or intravenous antibiotics.

Increasingly, dog bites are the subject of litigation because bite wounds are still being sutured when they should be left open and because of incorrect antimicrobial prophylaxis.

The "hole and tear" effect-whereby canine teeth anchor the person while other teeth bite, shear, and tear the tissues-results in stretch lacerations, easily piercing immature cranial bones. The biting force of canine jaws varies with the breed, from $310 \mathrm{kPa}$ to nearly 31790 $\mathrm{kPa}$ in specially trained attack dogs. ${ }^{\mathrm{w} 1 \mathrm{w} 2}$ Large wounds, significant devitalisation, and high mortality can result, with the highest mortality in neonates (six times that in toddlers), who are usually bitten by household pets. ${ }^{2} 3$

This review is aimed at clinicians who deal with dog bites. The basic principles of wound management and indications for use of antimicrobials and rabies prophylaxis apply to clinicians in all countries, but the primary focus of this article will be the UK.

Overall, the clinical approach in the UK to management of dog bites is pragmatic and based largely on consensus opinion rather than firm evidence. The major basis for recommending co-amoxiclav is in-vitro sensitivity data of organisms related to dog bites, and most authorities recommend using

Box 1 | Points to consider during history and examination

\section{History}

- For travellers bitten abroad, assess risk of rabies and consider rabies prophylaxis

- Note immunocompromising factors, such as splenectomy, cirrhosis, ${ }^{\mathrm{w} 5}$ and steroid therapy ${ }^{9}$

- Note recent antibiotics (infection despite flucloxacillin or erythromycin makes superinfection with resistant organisms such as Pasteurella multocida likely)

\section{Examination}

- Children with facial or cranial bites need cervical immobilisation until cervical lesions are excluded

- Take careful documentation with diagrams of the wound (photographs may be useful)

- Assess size and depth of the wound, degree of crush injury and devitalised tissue, nerve or tendon damage, and involvement of bones and joints

- Full wound examination and debridement, with local or general anaesthetic if necessary

- Radiography is indicated to exclude embedded teeth or dental fragments, fractures, and bony damage, or in scalp wounds in children

\section{SUMMARY POINTS}

Wound management is as important as use of antimicrobials in preventing infection

Primary closure should be avoided in limb injuries where possible because of increased risk of infection

For patients considered to be at higher risk of infection, the prophylaxis of choice is co-amoxiclav

Erythromycin or flucloxacillin should never be used alone prophylactically as Pasteurella infection is usually resistant Infected wounds presenting within 12 hours of injury are usually due to Pasteurella multocida

Patients at particularly high risk of infection are immunosuppressed patients, particularly those with asplenia or cirrhosis or those who have had a mastectomy

prophylactic antimicrobials in selected patients at high risk of infection.

\section{Sources and selection criteria}

We reviewed the Cochrane Library and performed Medline searches to identify relevant systematic reviews on the management of dog bites, using the keywords "dog-bites", "reviews", "prophylaxis", and "treatment". We consulted personal archives, Clinical Evidence, and UK national NHS (Prodigy) guidelines.

\section{How big is the problem?}

Of the estimated 740 people per 100000 population bitten by dogs annually, ${ }^{4}$ a minority seek medical attention. Overall, 2.6/100 000 population need hospital admission. Half of all children are reportedly bitten by dogs at some time, boys more than girls. A recent telephone survey of 1184 families found that the annual incidence of bites in children aged under 15 years was $22 / 1000 .^{5}$

Accurate mortality figures are poorly documented in the medical literature and difficult to obtain. However, because deaths are newsworthy, the popular press reports are probably reliable indicators of the true number in the UK, and during the past five years, two to three cases a year have made headlines. In the United States annual mortality is 7.1/100 million population, with $57 \%$ of deaths occurring in children aged under 10 years.

\section{Why do dogs bite?}

Most attacks are apparently unprovoked, but dogs are not always to blame. Dogs resent being disturbed while 


\section{Box 2 | Procedures for initial wound management}

- Irrigate copiously, using tap water or normal saline ${ }^{\text {w3 } w 4}$

- Remove foreign bodies (teeth)

- Perform a thorough wound toilet and debridement where necessary ${ }^{\mathrm{w} 3 \mathrm{w} / 4}$

- Delay closure of the wound where possible

- Raise and immobilise the limb if the injury is associated with (or is likely to cause) swelling

- Give antibiotics, depending on the risk factors for infection

-With infected wounds, send pus or a deep wound swab for culture (in clinically uninfected wounds, swabbing is unhelpful)

- Review bites within 24-48 hours, especially if the bites need antimicrobial prophylaxis

- Although tetanus after animal bites is rare, all guidelines in common use advise tetanus prophylaxis, with immunoglobulin and toxoid to be administered to patients with a history of two or fewer immunisations

\section{Box 3 | Factors that increase risk of infection*}

Patient factors
- Alcoholism (increased susceptibility to Pasteurella infection ${ }^{9}$ )
- Cirrhosis, asplenia (increased risk of Capnocytophaga)
- Steroid therapy, rheumatoid arthritis, diabetes mellitus, and lymphoedema after
radiotherapy (all increase risk of Pasteurella infection ${ }^{9}$
Wound factors
- Wounds $>6$ hours old
- Devitalised tissue ${ }^{10}$
- Previously sutured wounds
- Full thickness wounds involving tendons, ligaments, and joints
- Bites on limbs, especially hands
*According to case reports and small reviews

Box 4 | Indications for referral to specialist care ${ }^{9}$

- If systemic manifestations of infection are present

- If bone, joints, or tendons are affected

- If hand bites are serious or the bites require reconstructive surgery

- If bites are cranial, especially in an infant

- If the patient has severe cellulitis or infection is refractory to oral therapy eating and dislike being threatened or feeling that their territory is being invaded, and they can be jealous of attention given to other family members.

There is much debate about which dogs attack humans the most. Most reviewers conclude that the higher risk animals include larger dogs, German shepherd dogs, pit bull terriers, Rottweilers, and chows, but all dogs should be considered dangerous; even smaller dogs such as Jack Russell terriers inflict severe bites. $^{6-8}$

\section{What are the medicolegal aspects of dog bites?}

Litigation associated with dog bites occurs at a steady rate in the UK-initiated by people attacked while walking or delivering mail; compensation claims are also made regularly against clinicians for alleged mismanagement of the original injury. An estimated 5000 postal workers seek medical help for bites annually in the UK.

Police can prosecute owners under the Dangerous Dogs Act 1991 (which makes ownership of certain breeds illegal), and magistrates have the power to have a dog put down. A civil claim against the owner for damages can be made under the Animal Act 1971. Adults have a three year limit in which to begin action, and "no win, no fee" legal firms already exploit this area of litigation. Compensation claims have varied, from a few thousand pounds to tens of thousands of pounds (and even hundreds of thousands for sportsmen whose career is affected by injury).

\section{How should dog bites be managed?}

Box 1 suggests how to take a history and do an examination in a patient presenting with a dog bite, and box 2 outlines initial management procedures. Where adequate debridement of deep penetrating wounds is not possible, it is common practice, although unsupported by strong evidence, to irrigate the wound with $250 \mathrm{ml}$ saline, using a 19 or 20 gauge needle or plastic intravenous catheter on a $35 \mathrm{ml}$ syringe. ${ }^{\text {w3 w4 }}$

Irrigation is particularly important if the dog is suspected of being rabid. Gentle debridement after irrigation is essential as irrigation alone may not remove the virus from wound edges; the wound should then be covered with a sterile dressing or a clean dry cloth.

Factors increasing the risk of infection are arbitrarily divided into patient and wound factors (box 3). Many studies involving small numbers of patients have suggested various predisposing factors. A larger observational study-of 769 sequential patients with dog bite wounds presenting to an emergency departmentfound that the strongest predictors for the development of infection were wound depth, need for surgical debridement, and being female. ${ }^{9}$ Box 4 indicates when referral for specialist care is necessary.

\section{Head and neck bites}

Unlike adults, in whom only $10 \%$ of bites involve the head and neck, most bites in children are to the head or face, with $76 \%$ affecting lips, nose, or cheeks. ${ }^{11}$

Exsanguination after carotid trauma is the major cause of death due to bites in children aged under 10 years, so with major trauma, resuscitation is the priority. Penetrating wounds of the neck and thoracic inlet are especially dangerous, and early angiography and exploration may be necessary. Avulsed body parts should be kept cool pending reattachment.

A complete physical examination, followed by intraoral examination to exclude cheek lacerations extending into the oral cavity, is necessary. Children with facial or cranial bites need cervical immobilisation until cervical lesions are excluded. Careful examination and appropriate imaging are necessary; a small scalp puncture wound may overlie intracranial injury and facial fractures.

Facial bites can often be closed primarily. ${ }^{11} 12$ Although rarely necessary, antibiotic prophylaxis decreases the risk of infection to $1 \% .^{12}$

\section{Extremity and hand bites}

Anatomically, the hand contains many small compartments, and there is a relative lack of soft tissues separating the skin from the bone and joint. Surgical debridement needs to be done by an experienced clinician. Overall, only a fifth of dog bites become infected, compared with $36 \%$ of hand bites, ${ }^{13}$ and loss of function can result from infection. Hence thorough documentation of the injury and nerves affected is necessary. With a strict protocol of vigorous debridement and irrigation the infection rate can be as low as $0.5 \%{ }^{\text {w4 }}$ Pus needs draining and preferably should be cultured (actual pus rather than a swab). Wounds on extremities should 
not be closed primarily, but left unsutured (with the limb raised and immobilised) and sutured only when considered clean and free of infection.

With adequate debridement and wound toilet, primary suturing may be possible. ${ }^{14}$ Severe bites require "second look" surgery 24-48 hours after initial radical debridement-to exclude residual dead tissue or infective focus.

\section{Which infections result from animal bites?}

Dog bite related infections are polymicrobial, predominantly Pasteurella and Bacteroides spp. Infected bites presenting less than 12 hours after injury are particularly likely to be infected with Pasteurella spp, whereas those presenting more than 24 hours after the event are likely to be predominantly infected with staphylococci or anaerobes.

Inform the laboratory of the nature of the wound, as routine laboratory methods may fail to isolate or identify more unusual organisms. ${ }^{15}$ Thirty per cent of infections thought to be penicillin sensitive Staphylococcus aureus are actually $S$ intermedius. ${ }^{\text {w6 }}$

Culturing aerobically alone or for less than 5-7 days may explain the paucity of pathogens reported in older studies, particularly anaerobes such as Prevotella, Porphyromonas, and Fusobacteria spp. ${ }^{15}$

Dog bite organisms often have strange names, the classic example being Capnocytophaga canimorsus (dysgonic fermenter type 2 or DF2). With nearly 100 reported cases, DF2 septicaemia is often mistaken for fulminant meningococcal disease. ${ }^{16}$ w5 Infection usually follows a trivial bite in patients with asplenia or cirrhosis. Typically, Gram negative rods are seen within polymorphs on peripheral blood films. ${ }^{16}$ w5 DF2 is
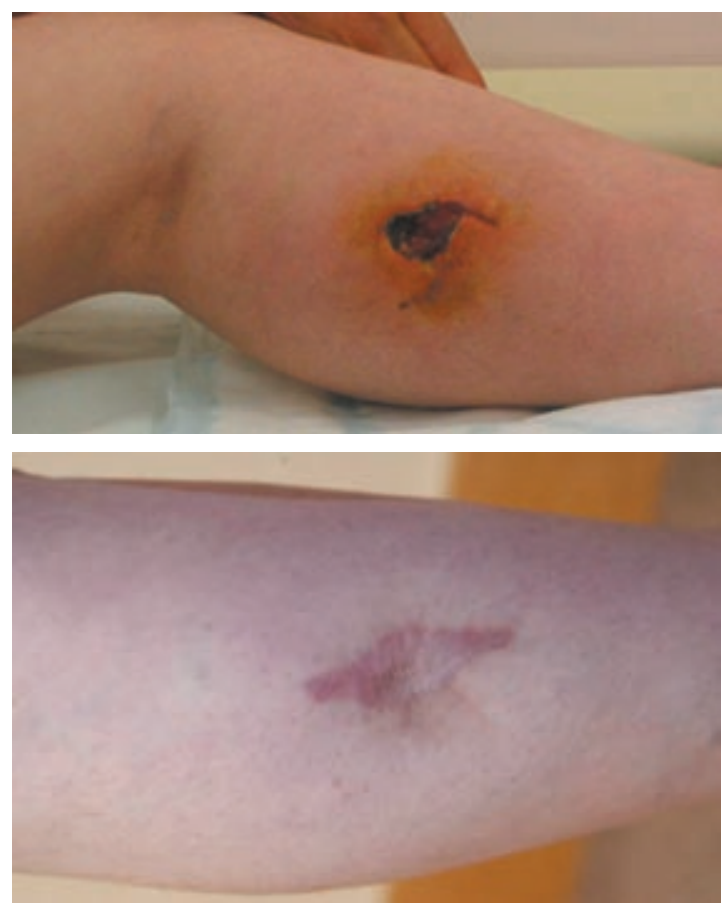

Fig 1 | Top: Breakdown of a bite wound and infection seven days after it was sutured in primary care. Bottom: Resultant scarring 18 months later

\section{Box 5 | Characteristics of Pasteurella multocida}

- Literally "killer of many species"-probably the most virulent pathogen in dog bites and responsible for severe infection

- Present in $>50 \%$ of dog bites ${ }^{15}$

- The most likely pathogen in infected wounds presenting within 12 hours of the bite ${ }^{15}$

- An aggressive Gram negative pathogen, causing early intense inflammatory response with considerable tissue involvement, and likely to cause metastatic infection with severe sequelae ${ }^{18}$

- Associated with a mortality of $30 \%$ in septicaemia ${ }^{18}$ w7

- Resistant to erythromycin and flucloxacillin ${ }^{17}$

- Likely to result in tenosynovitis in hand bites especially, and may lead to irreparable damage and amputation

sensitive to penicillin and ciprofloxacin.

Clinical infection may also result from incorrect management in primary care (figs 1 and 2). Erythromycin or flucloxacillin must never be used alone in prophylaxis. In one small study $70 \%$ patients with Pasteurella multocida infections (see box 5) had received inadequate or incorrect antibiotics, usually flucloxacillin or erythromycin. ${ }^{17}$ There are many reports of clinical failures and several deaths due to failure of erythromycin therapy. ${ }^{\mathrm{w} 7}$

\section{When should prophylactic antibiotics be used?}

As only a fifth of all dog bites become infected ${ }^{13}$ it is generally accepted that superficial, easily cleaned dog bite wounds do not warrant antibiotics if the patient is otherwise immunocompetent. We found no evidence justifying routine antibiotic prophylaxis for bites at low risk of infection. The consensus of opinion, however, is that antibiotic prophylaxis (co-amoxiclav) should be considered and is probably indicated for all "high risk" dog bites.

A postal survey of $21 \mathrm{UK}$ emergency departments and minor injury units found that prophylaxis was given routinely in 15 . Thirteen departments had a protocol, and co-amoxiclav was the antibiotic of first choice. ${ }^{\text {w8 }}$

No strong evidence base supports the routine use of co-amoxiclav. A series of methodologically poor studies, with differing dosages of various antimicrobials and inadequate microbiological methods, has produced a plethora of recommendations for prophylaxis with little valid evidence. The major basis for recommending co-amoxiclav is in-vitro sensitivity data.

The NHS guidelines (Prodigy) ${ }^{19}$ recommend coamoxiclav as first choice prophylaxis where indicated, since it covers all commonly expected organisms among the canine oral flora. ${ }^{\text {w9 }}$

Co-amoxiclav covers the penicillin resistant $S$ aureus and anaerobes and $P$ multocida, which is resistant to flucloxacillin and erythromycin. ${ }^{15}$ w10 w11

Some authors advise empirical prophylaxis for all animal bites, ${ }^{\text {w10 }}$ while others take a more sensible approach, restricting prophylaxis to injuries or patients deemed at high risk of infection. ${ }^{92122 \mathrm{w} 1 \mathrm{w} 3}$ 


\begin{tabular}{lll}
\hline $\begin{array}{l}\text { Meta-analyses of trials involving prophylactic antibiotics for dog bites } \\
\text { Study }\end{array}$ & $\begin{array}{l}\text { Conclusion } \\
\text { In four of the largest studies antibiotics } \\
\text { decreased the risk of infection; and to prevent } 1 \\
\text { infection, 14 patients needed prophylaxis }\end{array}$ & $\begin{array}{l}\text { Meta-analysis of } 8 \text { randomised trials; not } \\
\text { a systematic review; } 8 \text { trials, } 306 \text { patients; } \\
\text { different antibiotics compared, only } 1 \text { using co- } \\
\text { amoxiclav }\end{array}$ \\
\hline Cochrane review, $2001^{22}$ & No evidence of benefit in dog bites & $\begin{array}{l}8 \text { trials, including } 6 \text { randomised double blind } \\
\text { controlled and 1 randomised controlled trial; } \\
\text { different antibiotics compared, only } 1 \text { using co- } \\
\text { amoxiclav; small numbers of patients; different } \\
\text { antibiotic regimens; dog and other animal bites } \\
\text { included in trials }\end{array}$ \\
\hline
\end{tabular}

\section{Meta-analyses}

The conclusion and implications of two meta-analyses relating to antibiotic treatment for animal bites ${ }^{2122}$ are not directly relevant to UK practice (table). Both included trials with no stratification of risk of infection, comparing differing antibiotic regimens and dosages, ranging from penicillinase stable penicillins (such as oxacillin) to co-trimoxazole, cefalexin, and phenoxymethylpenicillin.

Each meta-analysis included eight trials, with six trials common to both. Cummings included one non-randomised controlled trial, ${ }^{21}$ and the Cochrane review included one trial of prophylaxis for cat bites and one for human bites. ${ }^{22}$ Overall, the patient numbers were small. Only one small trial involving 172 dog bites used co-amoxiclav. ${ }^{23}$ In that trial coamoxiclav (375 $\mathrm{mg}$ three times daily for five days) was given to 84 patients, with 88 given placebo. This resulted in a significant difference in infection rates $33 \%$ of those receiving co-amoxiclav prophylaxis became infected, compared with $60 \%$ receiving placebo)..$^{23}$

Most authors agree prophylaxis is of no proved benefit in simple facial dog bites, but the consensus of opinion recommends three to five days of prophylaxis for puncture wounds, ${ }^{9}{ }^{19}$ primary closures, ${ }^{11} 14$ high risk patients, and oral-cutaneous ("through and through") bites, ${ }^{24}$ with additional indications suggested by several authors of small reviews of treatment (box 6).

For patients with a true allergy to penicillin, effective alternatives to co-amoxiclav include tetracycline or doxycycline plus metronidazole, ${ }^{19}$ a second generation cephalosporin with anti-anaerobic activity such as ceftriaxone, or combination therapy with clindamycin and a fluoroquinolone.

Pregnant women with a history of rash after penicillin should be offered ceftriaxone.

\section{Treatment of established infection}

Inpatient treatment must cover Pasteurella, anaerobes, and staphylococci, and be modified according to culture results. For very severe infections, we use empirical imipenem with cilastatin $(500 \mathrm{mg}$ four times daily, intravenously) and clindamycin (900 mg four times daily, intravenously) until Gram stains or cultures are available to guide treatment. For patients with severe allergy to penicillin, ciprofloxacin (400 mg twice daily, intravenously) plus metronidazole (500 $\mathrm{mg}$ three times daily, intravenously) replaces imipenem.

\section{Duration of treatment for established infection}

In practice, treatment is usually 10-14 days for cellulitis, three weeks for tenosynovitis, four weeks for septic arthritis, and six weeks for osteomyelitis. Conversion to oral antibiotics when the $\mathrm{C}$ reactive protein concentration falls to $<50 \mathrm{mg} / \mathrm{l}$ is a pragmatic approach that we find works well in our hospital. If the $\mathrm{C}$ reactive protein levels off at a high concentration or continues to rise, then a clinical reappraisal is needed as a second debridement may be advisable, particularly with joint space infections.

\section{Rabies}

Rabies is transmitted by a transdermal bite or scratch, or salivary contamination of mucosa or skin wounds. It kills 30000 to 50000 people a year, mainly in

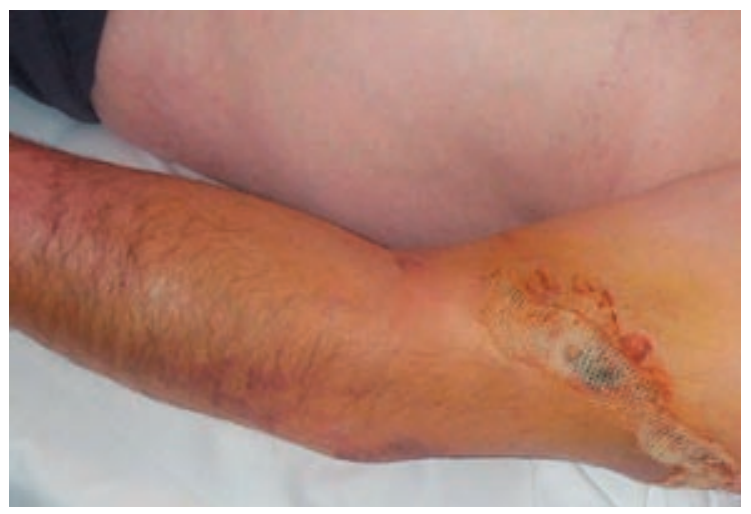

Fig 2 | Left: Bite laceration infected with Pasteurella multocida. Right: Haemorrhaging and resolving cellulitis in same patient

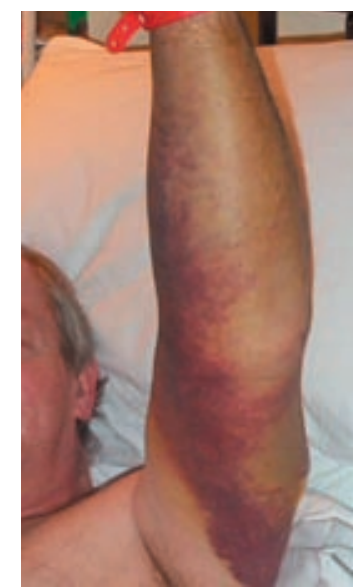

Box 6 | Indications for antimicrobial prophylaxis

"High risk" wounds

- All bite wounds after primary closure ${ }^{14}$

- Puncture wounds ${ }^{\text {w3 }}$

- Bites to hand and wrist ${ }^{\mathrm{w3}}$ w4

- Crush wounds with devitalised tissue ${ }^{9}$

- Dog bite injuries to the genitals ${ }^{10}$

"High risk" patients (conditions) ${ }^{9 \mathrm{w} 1}$

- Diabetes mellitus

- Immunosuppression

- Splenectomy, cirrhosis (C canimorsus)

- Postmastectomy ${ }^{w 1}$

- Rheumatoid arthritis ${ }^{\mathrm{w1}}$ and prosthetic joints ${ }^{\mathrm{w} 1} 2$ 
Box 7 | Who to contact about risk and management of rabies

- Health Protection Agency Centre for Infection, 61

Colindale Avenue, London NW9 5EQ www.hpa.org.uk

infections/default.htm

- Health Protection Scotland, Clifton House, Clifton Place, Glasgow G3 7LN www.hps.scot.nhs.uk,

developing countries and especially where unvaccinated stray dogs are common. Avoiding exposure to rabies involves education of travellers and advice not to touch animals abroad, especially if they appear unwell and have excessive salivation or paralysis. Prior rabies vaccination may be sensible for travellers to remote areas where rabies is highly endemic. ${ }^{25}$

Rabies is almost invariably fatal, so even seemingly minor bites in high risk countries should be taken seriously. Local medical advice should be sought on the risks of rabies and prophylaxis after exposure. Thorough cleansing significantly lessens the risk of rabies. Hence flushing the wound under a running tap for several minutes, washing with soapy water or detergent, and particularly using wound disinfectants (such as 40-70\% alcohol, tincture, or aqueous solution of povidone-iodine) is recommended. Again, primary suturing should be avoided if possible.

\section{Risk assessment in travellers returning with dog bites}

Rabies vaccine and immunoglobulin should be given if required. ${ }^{25}$ w13 Local advice should be sought, as countries differ in the risks of contracting rabies and in the administration and use of vaccine and immunoglobulin. For example, intradermal vaccination may be used in some countries where resources are scarce, and equine rabies immunoglobulin may be the only one available. For travellers returning home to the UK, intramuscular vaccine and human rabies immunoglobulin are obtained by contacting the centres listed in box 7 . Information that general practitioners will need to provide when discussing the need for prophylaxis with staff at the centres includes previous vaccination status, country where bitten, site and date of bite, provoked or unprovoked bite, domestic or feral dog, current health of animal, and previous immunisation status of patient.

\section{Prevention}

An educational intervention, "Prevent a bite" (designed primarily for schoolchildren), was effective in increasing precautionary behaviour among children when confronted with a dog. ${ }^{\text {w14 }}$ Generally, children should be taught to treat dogs with respect, avoid direct eye contact, and not tease them. They should be taught not to approach an unfamiliar dog; play with any dog unless under close supervision; run or scream in the presence of a dog; pet a dog without at first letting it sniff you; or disturb a dog that is eating, sleeping, or caring for puppies.

\section{We thank Elizabeth Saunders for valuable comments on the manuscript.}

Contributors: MM searched the literature on therapeutics and drafted the article; JP added aspects on surgery. Both authors completed and revised the content critically and are joint guarantors.

\section{ADDITIONAL EDUCATIONAL RESOURCES}

\section{Resources for healthcare professionals}

- Keogh S, Callaham M. Bites and injuries inflicted by domestic animals. In: Auerbach PS, ed. Wilderness medicine. 4th ed. St Louis, Missouri: Mosby, 2001.

- Solomon TN, Marston D, Mallewa M, Felton T, Shaw S, McElhinney LM, et al. Paralytic rabies after a two week holiday in India. BMJ 2005;331:501-3.

- Rupprecht CE, Hanlon CA, Hemachudha T. Rabies reexamined. Lancet Infect Dis 2002;2:327-43.

-Immunisation against infectious diseases-the green book. 2006 edition. www.dh.gov.uk/[search for title].

- Warrell MJ, Warrell DJ. Intradermal post exposure rabies vaccine regimens. Clin Infect Dis 2002;34:477-80.

Resources for patients

- Dog and cat bites www.prodigy.nhs.uk/patient information/pils/dog_and_cat_bites.pdfi)-guidance on what to do after a bite

- Travel health information sheet www.nathnac.org/travel/ factsheets/rabies1.htm-information about rabies

Competing interests: None declared.

Provenance: Commissioned, peer reviewed.

1 Thomas HF, Banks J. A survey of dog bites in Thanet.J R Soc Health 1990;110:173.

2 Sacks JJ, Lockwood R, Hornreich J, Sattin RW. Fatal dog attacks 1981-94. Pediatr 1996;9:891-5.

3 Rohrich RJ. Man's best friend revisited: who's watching the children? Plast Reconstr Surg 1999:2067-8

4 Wolff $K$. Management of animal bite injuries of the face: experience in 94 patients. J Oral Maxillofac Surg 1998;56:838-43.

5 De Keuster, T Lamoureaux J, Kahn A. Epidemiology of dog bites: a Belgian experience of canine behaviour and public health concerns. Vet / 2006;172:482-7.

6 Gershman KA, Sacks JJ, Wright JC. Which dogs bite? A case-control study of risk factors. Pediatrics 1994;93:913-7.

7 Shewell PC, Nancarrow JD. Dogs that bite. BMJ 1991;303:1512-3.

8 Cole RP. Dog bites to children. BMJ 1991;303:467.

9 Dire DJ, Hogan DO, Riggs MW. A prospective evaluation of risk factors from dog-bite wounds. Acad Emerg Med 1994;3:258-65.

10 Gomes CM, Ribeiro-Filho L, Giron AM, Mitre AL, Figueira ER, Arap S. Genital trauma due to animal bites. JUrol 2000;165:80-3.

11 Palmer J, Rees M. Dog bites of the face: a fifteen year review. Br J Plast Surg 1983;36:315-8.

12 McheikJN, Vergniss P, Bondonny JM. Treatment of facial dog bites injuries in children: a retrospective study. J Ped Surg 2000;35:580-3.

13 Callaham M. Controversies in antibiotic choices for bite wounds. Ann Emerg Med 1988;17:1321-9.

14 Moore F. I've just been bitten by a dog. BMJ 1997;314:88-9.

15 Talan DA, Citron DM, Abrahamian FM, Moran GJ, Goldstein EJC. Bacteriologic analysis of infected cat and dog bites. NEngl/ Med 1999;340:285-92.

16 Kullberg B-J, Westendorp RG, Van't Wout, JW Meinders AE. Purpura fulminans and symmetrical gangrene caused by Capnocytophaga canimorsus (formerly DF-2) septicaemia-a complication of dog bite. Medicine 1991;70:287-91.

17 Holm M, Tarnvik H. Hospitalisation due to Pasteurella multocidainfected animal bite wounds: correlation with inadequate primary antibiotic medication. Scand / InfectDis 2000;32:181-3.

18 Weber D, Wolfson JS, Swartz MN, Hooper DC. Pasteurella multocida infections: report of 34 cases and review of the literature. Medicine 1984;63:133-54.

19 Management of animal bites. Prodigy guidance. www.prodigy.nhs. uk/qrg/bites_animal.pdf

20 Griego RD, Rosen T, Orego IF, Woolf JE. Dog, cat and horse bites: a review. J Am Acad Dermatol 1995;33:1919-29.

21 Cummings P. Antibiotics to prevent infection in patients with dogbite wounds; a meta- analysis of randomised trials. Ann Emerg Med 1994;23:535-40.

22 Medeiros I, Saconato H. Antibiotic prophylaxis for mammalian bites. Cochrane Database Syst Rev 2001;(2):CD001738.

23 Brakenbury PH, Muwanga C. A comparative double-blind study of amoxycillin/clavulanate vs placebo in the prevention of infection after animal bites. Arch Emerg Med 1989;6:251-6.

24 Morgan JP, Haug RH, Murphy MT. Management of facial dog bite injuries. J Oral Maxillofac Surg 1995;53:435-41.

25 Pounder D. Avoiding rabies. BM/ 2005;331:469-70. 